\title{
Commutators of singular integrals, the Bergman projection, and boundary regularity of elliptic equations in the plane
}

\author{
Alexander Tumanov
}

We obtain estimates of commutators of singular integral operators in Lipschitz spaces and apply the results to boundary regularity of elliptic equations in the plane. We obtain an explicit asymptotic formula for the Bergman projection.

\section{Introduction}

In this paper we are concerned with sharp boundary regularity in Lipschitz spaces $C^{k, \alpha}$ of first order elliptic equations of the form

$$
f_{\bar{z}}=a(z) f_{z}+b(z) \bar{f}_{\bar{z}}+c(z)
$$

in a smooth bounded domain $\Omega$ in complex plane $\mathbb{C}$. Here $f_{z}=\partial f / \partial z$ and $f_{\bar{z}}=\partial f / \partial \bar{z}$. We impose the ellipticity condition

$$
|a(z)|+|b(z)| \leq a_{0}<1
$$

for some constant $a_{0}$. We first consider the scalar equation (1) with the Dirichlet type boundary condition Re $\left.f\right|_{b \Omega}=f_{0}$ for given function $f_{0}$ on the boundary. Suppose $a, b, c$ are in $C^{k, \alpha}(\Omega), k \geq 0,0<\alpha<1$. Suppose $f_{0}$ is in $C^{k+1, \alpha}(b \Omega)$. We would like to conclude that every generalized solution of (1) with $\left.\operatorname{Re} f\right|_{b \Omega}=f_{0}$ is automatically in $C^{k+1, \alpha}(\Omega)$. Apparently, this classical question is not covered in the extensive literature on the subject. In particular, the case of first order equations does not follow from the classical results on boundary regularity of elliptic equations $[1,11]$. If in the

2010 Mathematics Subject Classification: 35J56, 42B20, 30H20.

Key words and phrases: elliptic equation, commutator, singular integral, Bergman projection. 
scalar equation (1), the coefficient $b=0$, then the conclusion is rather simple (see [12], Proposition 2.1). Tadeusz Iwaniec explained to the author that for $k \geq 1$ the Equation (1) can be reduced to a second order equation, and then the conclusion follows from Schauder's theory [11]. In this paper we give a proof for all $k \geq 0$. We also consider the vector version of (1) and give a proof of the regularity of the Dirichlet problem for $a=0$.

Another common boundary condition for Equation (1) is $K_{\Omega} f=f_{0}$. Here $K_{\Omega}$ is the Cauchy type integral (15) and $f_{0}$ is a given holomorphic function in $\Omega$. In particular, the homogeneous condition $K_{\Omega} f=0$ means that $f$ holomorphically extends to $\mathbb{C} \backslash \Omega$ and vanishes at infinity. Solving (1) with this boundary condition is equivalent to the problem of inverting the operator $f \mapsto f-T_{\Omega}\left(a f_{z}+b \bar{f}_{\bar{z}}\right)$, here $T_{\Omega}$ is the Cauchy-Green operator (14). The vector version of this problem with $a=0$ and small $b$ arises in constructing small pseudoholomorphic curves (see $[4,7,9]$ ). We prove the boundary regularity of this problem in the scalar case for general $a$ and $b$ satisfying (2) and in the vector case for $a=0$ and $\|b\|_{\infty}<1$, answering a question raised in $[4]$.

A classical approach $[2,13]$ to Equation (1), in particular, the Beltrami equation consists of reducing (1) to an integral equation with the operator $S_{\Omega}$ given by $(3)$ or its modifications. The solution operator of the integral equation is bounded in $L^{p}(\Omega)$ for $p$ close to 2 . In this approach, it is essential that $\left\|S_{\Omega}\right\|_{2} \leq 1$. However, there is more precise information about the operator $S_{\Omega}$, in particular, $S_{\mathbb{C}}$ is an isometry of $L^{2}(\mathbb{C})$, that is, $S_{\mathbb{C}} \bar{S}_{\mathbb{C}}=I$. There is a related property of $S_{\Omega} \bar{S}_{\Omega}$ that we derive in Section 4. An iteration of the integral equation corresponding to (1) with $b \neq 0$ involves the term $S_{\Omega} b \bar{S}_{\Omega} \bar{b}$, here $b$ denotes the operator of multiplication by $b$. Since $S_{\Omega}$ and $\bar{S}_{\Omega}$ do not stand next to each other, in order to make use of $S_{\Omega} \bar{S}_{\Omega}$ we need information about the commutators of $S_{\Omega}$ with multiplication operators, namely, their smoothing properties.

There are well known $L^{p}$ estimates of commutators of singular integral operators with multiplication operators (see, e. g., [5, 6]). However, apparently, $C^{k, \alpha}$ estimates of the commutators are covered in the literature only for the case of Cauchy type integrals and similar operators (see [10], Section 3.4.1). We present results on the matter for the operator $S_{\Omega}$. Although we use complex variable notations, the results are real in nature and could be established for more general Calderón-Zigmund operators.

As we mentioned above, the scalar equation (1) with $b=0$ is rather simple. If $b=0$, then the Equation (1) can be reduced to the case $a=b=0$ by changing the independent variable. Our method involving $S_{\Omega} \bar{S}_{\Omega}$ and the commutators now lets us deal with the case $a=0, b \neq 0$. In the scalar case, 
it suffices for treating the general equation (1) because we can reduce it to $a=0$. However, in the vector case, obviously, the reduction to $a=0$ by changing the independent variable is not possible in general, thus we only handle the vector equation (1) for $a=0$.

In Sections 2 and 3 we include results on $C^{k, \alpha}$ regularity of commutators of $S_{\Omega}$ with multiplication operators. In Section 4 we study properties of $S_{\Omega} \bar{S}_{\Omega}$. In Section 5 we give an asymptotic formula of the Bergman projection for $\Omega$ in terms of $S_{\Omega} \bar{S}_{\Omega}$. In Section 6 we treat integral equations corresponding to the vector version of the Equation (1) with $a=0$. Finally, in Sections 7 and 8 we study the boundary regularity of the Equation (1).

\section{Commutators of singular integrals}

For a domain $\Omega \subset \mathbb{C}$, we consider the Calderón-Zigmund operator (see $[2$, 13])

$$
S_{\Omega} u(z)=\mathrm{p} \cdot \mathrm{v} \cdot \int_{\Omega} \frac{u(t) d^{2} t}{(t-z)^{2}}
$$

Here for brevity $d^{2} t=(2 \pi i)^{-1} d t \wedge d \bar{t}$, and the integral is understood as Cauchy principal value. Let $a(z)$ be a function in $\Omega$. We use the same notation $a$ for the operator of multiplication by $a$. We are concerned with smoothing properties of the commutator

$$
\left[S_{\Omega}, a\right] u(z)=\int_{\Omega} \frac{a(t)-a(z)}{(t-z)^{2}} u(t) d^{2} t
$$

in Lipschitz spaces. As usual $C^{k, \alpha}(\Omega)$ denotes the space of functions whose derivatives to order $k \geq 0$ satisfy a Lipschitz condition with exponent $0<$ $\alpha<1$. We also sometimes write $C^{\alpha}(\Omega)=C^{0, \alpha}(\Omega)$ and $C^{k+\alpha}(\Omega)=C^{k, \alpha}(\Omega)$. We do not make a difference between $C^{k, \alpha}(\Omega)$ and $C^{k, \alpha}(\bar{\Omega})$. If $k$ is integer, then we use $C^{k}(\Omega)$ for the usual $C^{k}$-smooth functions. We use $C^{k, \alpha}(\Omega, \mathbb{R})$ for the set of real valued functions in $C^{k, \alpha}(\Omega)$. We denote by $L_{R}^{p}$ and $C_{R}^{k, \alpha}$ the spaces of functions respectively in $L^{p}(\mathbb{C})$ and $C^{k, \alpha}(\mathbb{C})$ with support in the disc $|z| \leq R$.

Theorem 2.1. Let $0<\alpha<1,0<\beta<\alpha, R>0$. Let $S=S_{\mathbb{C}}$.

(i) If $a \in C^{\alpha}(\mathbb{C})$, then the commutator $[S, a]$ is a bounded operator $L_{R}^{\infty} \rightarrow$ $C^{\beta}(\mathbb{C})$ and $C_{R}^{\beta} \rightarrow C^{\alpha}(\mathbb{C})$. 
(ii) If $a \in C^{k+1, \alpha}(\mathbb{C}), k \geq 0$, then $[S, a]$ is a bounded operator $C_{R}^{k, \alpha} \rightarrow$ $C^{k+1, \alpha}(\mathbb{C})$.

We begin the proof with a simple formula. We introduce the difference and shift operators

$$
\Delta_{h} f(z)=f(z+h)-f(z), \quad \delta_{h} f(z)=f(z+h) .
$$

Lemma 2.2. For the operator $S=S_{\mathbb{C}}$, the following formula holds.

$$
\Delta_{h}[S, a] u=\left[S, \Delta_{h} a\right] \delta_{h} u+[S, a] \Delta_{h} u .
$$

Proof. Clearly $\left[\Delta_{h}, S\right]=0,\left[\delta_{h}, S\right]=0$, and $\left[\Delta_{h}, a\right]=\left(\Delta_{h} a\right) \delta_{h}$. Then

$$
\begin{aligned}
\Delta_{h}[S, a] & =\Delta_{h} S a-\Delta_{h} a S=S \Delta_{h} a-\Delta_{h} a S \\
& =S\left(\left[\Delta_{h}, a\right]+a \Delta_{h}\right)-\left(\left[\Delta_{h}, a\right]+a \Delta_{h}\right) S \\
& =S\left(\left(\Delta_{h} a\right) \delta_{h}+a \Delta_{h}\right)-\left(\left(\Delta_{h} a\right) \delta_{h}+a \Delta_{h}\right) S \\
& =\left[S\left(\Delta_{h} a\right) \delta_{h}-\left(\Delta_{h} a\right) S \delta_{h}\right]+\left[S a \Delta_{h}-a S \Delta_{h}\right] \\
& =\left[S, \Delta_{h} a\right] \delta_{h}+[S, a] \Delta_{h} .
\end{aligned}
$$

Proof of Theorem 2.1. (i) Without loss of generality we can assume $a(z)$ has compact support because if $a(z)=0$, say for $|z| \leq 2 R$, then the result is obvious.

Let $f=[S, a] u$. We will write $C_{1}, C_{2}, \ldots$ for constants that may depend on $\alpha, \beta$, and $R$. First of all

$$
|f(z)| \leq\|a\|_{C^{\alpha}}\|u\|_{\infty} \int_{|t|<R}|t-z|^{\alpha-2}\left|d^{2} t\right| .
$$

Hence $\|f\|_{\infty} \leq C_{1}\|a\|_{C^{\alpha}}\|u\|_{\infty}$. Define

$$
K(z, t)=K(z)(t)=\frac{a(t)-a(z)}{(t-z)^{2}} .
$$

In estimating $\Delta_{h} f(z)$ for simplicity put $z=0$. Introduce

$$
F(t)=\Delta_{h} K(0)(t)=\frac{a(t)-a(h)}{(t-h)^{2}}-\frac{a(t)-a(0)}{t^{2}} .
$$

For $|t|<2|h|$ we use the estimate

$$
|F(t)| \leq\|a\|_{C^{\alpha}}\left(|t-h|^{\alpha-2}+|t|^{\alpha-2}\right) .
$$


For $|t|>2|h|$ we rewrite $F(t)$ in the form

$$
F(t)=\frac{h(2 t-h)(a(t)-a(h))}{t^{2}(t-h)^{2}}-\frac{a(h)-a(0)}{t^{2}},
$$

in which the first term does not exceed $C_{2}\|a\|_{C^{\alpha}}|t|^{\alpha-3}|h|$.

We have $\Delta_{h} f(0)=\int_{\mathbb{C}} F(t) u(t) d^{2} t=J_{1}+J_{2}-(a(h)-a(0)) J_{3}$. Here

$$
\begin{aligned}
J_{1} & =\int_{|t|<2|h|} F(t) u(t) d^{2} t, \\
J_{2} & =\int_{|t|>2|h|} \frac{h(2 t-h)(a(t)-a(h))}{t^{2}(t-h)^{2}} u(t) d^{2} t, \\
J_{3} & =\int_{|t|>2|h|} \frac{u(t)}{t^{2}} d^{2} t .
\end{aligned}
$$

Then $J_{1}$ and $J_{2}$ admit the following similar estimates

$$
\begin{aligned}
& \left|J_{1}\right| \leq\|a\|_{C^{\alpha}}\left|\int_{|t|<2|h|}\left(|t-h|^{\alpha-2}+|t|^{\alpha-2}\right) u(t) d^{2} t\right| \leq C_{3}\|a\|_{C^{\alpha}}\|u\|_{\infty}|h|^{\alpha}, \\
& \left|J_{2}\right| \leq\left.\left. C_{4}\|a\|_{C^{\alpha}}|h|\left|\int_{|t|>2|h|}\right| t\right|^{\alpha-3} u(t) d^{2} t\left|\leq C_{5}\|a\|_{C^{\alpha}}\|u\|_{\infty}\right| h\right|^{\alpha} .
\end{aligned}
$$

Let $u \in L_{R}^{\infty}$. Then $J_{3}$ has the obvious estimate

$$
\left|J_{3}\right| \leq\|u\|_{\infty} \int_{2|h|<|t|<R}|t|^{-2}\left|d^{2} t\right| \leq C_{6} \log |h|^{-1}\|u\|_{\infty} .
$$

Hence $\left\|\Delta_{h} f\right\|_{\infty} \leq C_{7}\|a\|_{C^{\alpha}}\|u\|_{\infty}|h|^{\beta}$ and $f \in C^{\beta}(\mathbb{C})$, which completes the proof of the first assertion in part (i).

Let $u \in C_{R}^{\beta}$. Since $S$ is bounded in $C^{\beta}$,

$$
\begin{aligned}
\left|J_{3}\right| & =\left|S u(0)-\int_{|t|<2|h|} \frac{u(t)-u(0)}{t^{2}} d^{2} t\right| \\
& \leq|S u(0)|+C_{6}\|u\|_{C^{\beta}}|h|^{\beta} \leq C_{7}\|u\|_{C^{\beta}} .
\end{aligned}
$$

Hence $f \in C^{\alpha}(\mathbb{C})$, which completes the proof of (i).

(ii) Let $k=0, a \in C^{1, \alpha}(\mathbb{C}), u \in C_{R}^{\alpha}$, and $f=[S, a] u$. We estimate the second difference $\Delta_{h}^{2} f$. It suffices to show $\left|\Delta_{h}^{2} f\right| \leq C_{8}\|a\|_{C^{1, \alpha}}\|u\|_{C^{\alpha}}|h|^{1+\alpha}$. 
By Lemma 2.2

$$
\Delta_{h}^{2} f=\Delta_{h}\left[S, \Delta_{h} a\right] \delta_{h} u+\Delta_{h}[S, a] \Delta_{h} u
$$

Consider the first term $A_{1}=\Delta_{h}[S, b] v$, here $b=\Delta_{h} a, v=\delta_{h} u$. Then by the same method we obtain $\left|A_{1}(0)\right| \leq C_{8}\|b\|_{C^{\alpha}}\|v\|_{C^{\alpha}}|h|^{\alpha}$. Clearly $\|b\|_{C^{\alpha}} \leq$ $C_{9}\|a\|_{C^{1, \alpha}}|h|$ and $\|v\|_{C^{\alpha}}=\|u\|_{C^{\alpha}}$. Hence $\left\|A_{1}\right\|_{\infty} \leq C_{10}\|a\|_{C^{1, \alpha}}\|u\|_{C^{\alpha}}|h|^{1+\alpha}$.

The second term $A_{2}=\Delta_{h}[S, a] v$ is more involved. Here $v=\Delta_{h} u,\|v\|_{\infty} \leq$ $\|u\|_{C^{\alpha}}|h|^{\alpha}$. Using the same notation as above, we write $\left|A_{2}(0)\right| \leq\left|J_{1}\right|+$ $\left|J_{2}\right|+\|a\|_{C^{1, \alpha}}\left|h J_{3}\right|$. The terms $J_{1}$ and $J_{3}$ are handled in the same manner as above; they admit the desired estimate. In particular,

$$
\begin{aligned}
\left|J_{3}\right| & =\left|\int_{|t|>2|h|} \frac{\Delta_{h} u(t)}{t^{2}} d^{2} t\right| \\
& =\left|\Delta_{h} S u(0)-\int_{|t|<2|h|} \frac{\Delta_{h} u(t)-\Delta_{h} u(0)}{t^{2}} d^{2} t\right| \leq C_{11}\|u\|_{C^{\alpha}}|h|^{\alpha} .
\end{aligned}
$$

We rewrite the remaining term $J_{2}=J_{4}+J_{5}$ as a result of splitting the factor $(2 t-h)$ in (6) into the sum $2 t-h=h+2(t-h)$. Then

$$
J_{4}=h^{2} \int_{|t|>2|h|} \frac{a(t)-a(h)}{t^{2}(t-h)^{2}} v(t) d^{2} t, \quad J_{5}=2 h \int_{|t|>2|h|} \frac{a(t)-a(h)}{t^{2}(t-h)} v(t) d^{2} t .
$$

Since $\|v\|_{\infty} \leq\|u\|_{C^{\alpha}}|h|^{\alpha}$, the integral $J_{4}$ admits a simple estimate

$$
\left|J_{4}\right| \leq\|a\|_{C^{1, \alpha}}\|u\|_{C^{\alpha}}|h|^{2+\alpha} \int_{|t|>2|h|}|t|^{-3}\left|d^{2} t\right| \leq C_{12}\|a\|_{C^{1, \alpha}}\|u\|_{C^{\alpha}}|h|^{1+\alpha} .
$$

For the remaining term $J_{5}$, we use Taylor's formula

$$
a(t)-a(z)=a_{z}(z)(t-z)+a_{\bar{z}}(z) \overline{(t-z)}+O\left(|t-z|^{1+\alpha}\right) .
$$

Then $J_{5}=2 h\left(a_{z}(h) J_{6}+a_{\bar{z}}(h) J_{7}+J_{8}\right)$, here

$$
J_{6}=\int_{|t|>2|h|} \frac{\Delta_{h} u(t) d^{2} t}{t^{2}}, \quad J_{7}=\int_{|t|>2|h|} \frac{\overline{t-h}}{\frac{\Delta_{h} u(t) d^{2} t}{t^{2}}},
$$

and $J_{8}$ comes from the remainder in (7). The term $J_{8}$ has the order $|h|^{2 \alpha}$, which is even better that we need. The term $J_{6}$ is the same as $J_{3}$ above. Hence the desired result for the commutator $[S, a] u$ is equivalent to the 
estimate

$$
\left|J_{7}\right| \leq C_{13}\|u\|_{C^{\alpha}}|h|^{\alpha}
$$

which is independent of $a$. Instead of dealing with $J_{7}$ directly, we observe that (8) is equivalent to the desired result for $[S, a] u$ with $a(z)=\bar{z}$. In this case the commutator turns into an integral similar to the well known CauchyGreen operator (14), for which the needed result is well known (see $[2,13])$. This remark completes the proof of (ii) for $k=0$.

We now consider $k>0$. By induction we assume that the result is already known for lower values of $k$. Let $D_{h} u(z)=\left.\frac{d}{d t}\right|_{t=0} u(z+t h)$ denote the directional derivative of $u$ in the direction $h$. Let $a \in C^{k+1, \alpha}$ and $u \in C_{R}^{k, \alpha}$. Then the result obtained for $k=0$ lets us pass to the limit in (5) to obtain

$$
D_{h}[S, a] u=\left[S, D_{h} a\right] u+[S, a] D_{h} u .
$$

Now by induction the result holds for all $k \geq 0$. The proof of Theorem 2.1 is complete.

Remark 2.3. The commutator $[S, a]$ in Theorem 2.1(i) is in fact a bounded operator $L_{R}^{p} \rightarrow C^{\beta}(\mathbb{C})$ for $p=\frac{2}{\alpha-\beta}$. Indeed, along the lines of the above proof one can show that $\|K(z)\|_{q} \leq C\|a\|_{C^{\alpha}}$ and $\left\|\Delta_{h} K(z)\right\|_{q} \leq C\|a\|_{C^{\alpha}}|h|^{\beta}$, here $\frac{1}{p}+\frac{1}{q}=1$. Then the conclusion follows by Hölder inequality.

\section{Commutators in a bounded domain}

We extend the result of the previous section to a bounded domain.

Theorem 3.1. Let $\Omega \subset \mathbb{C}$ be a bounded domain of class $C^{1, \alpha}, 0<\alpha<1$.

(i) If $a \in C^{\alpha}(\Omega)$, then for every $0<\beta<\alpha$, the commutator $\left[S_{\Omega}, a\right]$ is a bounded operator $L^{\infty}(\Omega) \rightarrow C^{\beta}(\Omega)$ and $C^{\beta}(\Omega) \rightarrow C^{\alpha}(\Omega)$.

(ii) If $\Omega$ and $a(z)$ are smooth of class $C^{k+1, \alpha}, k \geq 0$, then the commutator $\left[S_{\Omega}, a\right]$ is a bounded operator $C^{k, \alpha}(\Omega) \rightarrow C^{k+1, \alpha}(\Omega)$.

Taking into account Remark 2.3, the commutator $\left[S_{\Omega}, a\right]$ in (i) is in fact a bounded operator $L^{p}(\Omega) \rightarrow C^{\beta}(\Omega)$ for $p=\frac{2}{\alpha-\beta}$.

We first recall some simple estimates. Denote by $s=\operatorname{dist}(z, b \Omega)$ the distance from $z$ to $b \Omega$. Let $r, n \geq 0$ be integers. Introduce

$$
Q_{n}^{r} u(z)=\int_{\mathbb{C} \backslash \Omega} \frac{(\bar{t}-\bar{z})^{r} u(t) d t \wedge d \bar{t}}{(t-z)^{n}}
$$


Lemma 3.2. Let $\Omega \subset \mathbb{C}$ be a bounded domain of class $C^{k+1, \alpha}, k \geq 0,0<$ $\alpha<1$. Let $u \in C^{k, \alpha}(\mathbb{C})$. Then there is a constant $C>0$ depending on $\Omega, k$, and $\alpha$ so that for $z \in \Omega$

$$
\left|Q_{n}^{r} u(z)\right| \leq \begin{cases}C\|u\|_{C^{k, \alpha}} & \text { if } 3 \leq n-r<k+3 \\ C\|u\|_{C^{k, \alpha}} s^{\alpha-1} & \text { if } n-r=k+3 .\end{cases}
$$

Proof. Using induction on $k$, let $k=0, n-r=3$. Then

$$
Q_{n}^{r} u(z)=\int_{\mathbb{C} \backslash \Omega} \frac{(\bar{t}-\bar{z})^{r}(u(t)-u(z)) d t \wedge d \bar{t}}{(t-z)^{n}}+u(z) Q_{n}^{r}(1)(z),
$$

in which the first term clearly admits the estimate $O\left(s^{\alpha-1}\right)$ by integrating the modulus of the integrand. The second term will be automatically considered simultaneously with the general case.

Now let $k \geq 1$ and assume the estimate (9) for lower values of $k$. We also allow $k=0, u \equiv 1$. Let $b \Omega$ be a level set of a function of class $C^{k+1, \alpha}$. Then on $b \Omega$ we have $d \bar{t}=\phi(t) d t$, here $\phi \in C^{k, \alpha}$. We assume $\phi$ extends to the whole plane and has compact support. Introduce

$$
K_{m}^{r} u(z)=\int_{b \Omega} \frac{(\bar{t}-\bar{z})^{r} u(t) d t}{(t-z)^{m}}
$$

By Stokes' formula

$$
K_{n-1}^{r}(u \phi)=(n-1) Q_{n}^{r} u+Q_{n-1}^{r} u_{z}
$$

The term $Q_{n-1}^{r} u_{z}$ satisfies (9) by induction. Hence it suffices to show that $K_{n-1}^{r}(u)$ satisfies $(9)$ for $u \in C^{k, \alpha}(\mathbb{C}), k \geq 0$.

Integrating by parts for $m>1$ yields

$$
(1-m) K_{m}^{r} u=r K_{m-1}^{r-1}(u \phi)+K_{m-1}^{r}\left(u_{z}+u_{\bar{z}} \phi\right)
$$

Starting with $m=n-1$, we successively integrate by parts all resulting terms while still possible. If $n-r<k+3$, then all final terms will have the form $K_{m}^{p}(\psi)$ with $m<p+2, \psi \in C^{\alpha}$. They are clearly bounded. If $n-r=$ $k+3$, then all final terms will have the form $K_{p+2}^{p}(\psi)$ with $\psi \in C^{\alpha}$. We have

$$
K_{p+2}^{p}(\psi)(z)=\int_{b \Omega} \frac{(\bar{t}-\bar{z})^{p}(\psi(t)-\psi(z)) d t \wedge d \bar{t}}{(t-z)^{p+2}}+\psi(z) K_{p+2}^{p}(1)(z),
$$


The first term clearly has the estimate $O\left(s^{\alpha-1}\right)$. For the second one, we again reduce the exponent $p$ by integration by parts and eventually obtain the same estimate $O\left(s^{\alpha-1}\right)$.

Proof of Theorem 3.1. (i) The assertion about the map $L^{\infty}(\Omega) \rightarrow C^{\beta}(\Omega)$ follows immediately from the corresponding assertion of Theorem 2.1 because $a \in C^{\alpha}(\Omega)$ can be extended to a function of $C^{\alpha}(\mathbb{C})$, and for $R$ large enough, $L^{\infty}(\Omega) \subset L_{R}^{\infty}$ by trivial extension. So we focus on $\left[S_{\Omega}, a\right]$ on the space $C^{\beta}(\Omega)$.

We again consider the extension of $a \in C^{\alpha}(\Omega)$ to the whole plane (which we still denote by the same letter $a$ ) that has compact support, smooth in $\mathbb{C} \backslash \bar{\Omega}$, and whose first derivatives admit the estimate $O\left(s^{\alpha-1}\right)$. Similarly, we extend the restriction $\left.a\right|_{b \Omega}$ inside $\Omega$ so that the extension $\tilde{a}$ is smooth in $\Omega$ with first derivatives of the magnitude $O\left(s^{\alpha-1}\right)$. Finally, we extend the given function $u \in C^{\beta}(\Omega)$ to the whole plane so that the extension has compact support and belongs to $C^{\beta}(\mathbb{C})$. Then for $z \in \Omega$,

$$
\begin{aligned}
& {\left[S_{\Omega}, a\right] u(z)=\left[S_{\mathbb{C}}, a\right] u(z)-v_{1}(z)-v_{2}(z),} \\
& v_{1}(z)=\int_{\mathbb{C} \backslash \Omega} \frac{a(t)-\tilde{a}(z)}{(t-z)^{2}} u(t) d^{2} t, \quad v_{2}(z)=\int_{\mathbb{C} \backslash \Omega} \frac{\tilde{a}(z)-a(z)}{(t-z)^{2}} u(t) d^{2} t
\end{aligned}
$$

By Theorem 2.1, the first term $\left[S_{\mathbb{C}}, a\right] u$ has the desired properties. Differentiating $v_{1}$ yields

$$
\left(v_{1}\right)_{z}(z)=-\tilde{a}_{z}(z) \int_{\mathbb{C} \backslash \Omega} \frac{u(t) d^{2} t}{(t-z)^{2}}+2 \int_{\mathbb{C} \backslash \Omega} \frac{a(t)-\tilde{a}(z)}{(t-z)^{3}} u(t) d^{2} t .
$$

For $u \in C^{\beta}$ the first integral in (11) is bounded. Since $\tilde{a}_{z}=O\left(s^{\alpha-1}\right)$, the first term in (11) is $O\left(s^{\alpha-1}\right)$.

The second integral in (11) has the estimate $O\left(\int_{|t|>s}|t|^{\alpha-3}\left|d^{2} t\right|\right)=$ $O\left(s^{\alpha-1}\right)$. The $\bar{z}$-derivative of $v_{1}$ is estimated similarly but slightly simpler. Then by Hardy-Littlewood lemma, $v_{1} \in C^{\alpha}(\Omega)$.

We now consider $v=v_{2}=b w$. Here $b=\tilde{a}-a \in C^{\alpha}(\Omega),\left.b\right|_{b \Omega}=0, b=$ $O\left(s^{\alpha}\right) ; w=S_{\mathbb{C} \backslash \Omega} u$. Let $z, z^{\prime} \in \Omega$; without loss of generality $z$ is closer to $b \Omega$ than $z^{\prime}$. We estimate $\Delta v=v(z)-v\left(z^{\prime}\right)$ in terms of $h=\left|z-z^{\prime}\right|$. We have

$$
\Delta v=\Delta b w(z)+b\left(z^{\prime}\right) \Delta w
$$

Since $u \in C^{\beta}(\mathbb{C})$, we have $w(z)=O(1), w_{z}(z)=O\left(s^{-1}\right), \Delta w=O\left(h s^{-1}\right)$. Let $h \leq s$. Then $\Delta b=O\left(h^{\alpha}\right), b\left(z^{\prime}\right)=O\left(s^{\alpha}\right)$. Plugging these estimates in (12) 
yields

$$
\Delta v=O\left(h^{\alpha}+s^{\alpha} h s^{-1}\right)=O\left(h^{\alpha}\right) .
$$

If $h \geq s$, then $v(z)=O\left(s^{\alpha}\right), v\left(z^{\prime}\right)=O\left(h^{\alpha}\right)$, and again $\Delta v=O\left(h^{\alpha}\right)$. Hence $\left[S_{\Omega}, a\right] u \in C^{\alpha}(\Omega)$ as desired.

(ii) Let $a \in C^{k+1, \alpha}(\Omega)$. We again assume that $a(z)$ and $u(z)$ are extended to the whole plane. (We do not need the other extension $\tilde{a}$.) We represent

$$
\begin{aligned}
& {\left[S_{\Omega}, a\right] u=\left[S_{\mathbb{C}}, a\right] u-v, \quad v=\left[S_{\mathbb{C} \backslash \Omega}, a\right] u,} \\
& v(z)=\int_{\mathbb{C} \backslash \Omega} \frac{a(t)-a(z)}{(t-z)^{2}} u(t) d^{2} t .
\end{aligned}
$$

By Theorem 2.1, $\left[S_{\mathbb{C}}, a\right] u \in C^{k+1, \alpha}(\Omega)$. To see that $v \in C^{k+1, \alpha}(\Omega)$, we first differentiate it $(k+1)$ times. One term will have the form

$$
-D^{k+1} a S_{\mathbb{C} \backslash \Omega} u,
$$

here $D^{k+1} a$ denotes any derivative of order $(k+1)$. This term is clearly in $C^{\alpha}(\Omega)$. To show that the other terms are in $C^{\alpha}(\Omega)$, we show that the first derivatives of these terms have the estimate $O\left(s^{\alpha-1}\right)$. Then by HardyLittlewood lemma we will obtain $v \in C^{k+1, \alpha}(\Omega)$. By differentiating one more time, we obtain the following terms. There will be one term of the form

$$
J_{1}(z)=\int_{\mathbb{C} \backslash \Omega} \frac{a(t)-a(z)}{(t-z)^{k+4}} u(t) d^{2} t,
$$

while all other terms will be constant multiples of integrals of the form

$$
J_{2}(z)=D^{p} a(z) \int_{\mathbb{C} \backslash \Omega} \frac{u(t) d^{2} t}{(t-z)^{q+2}} .
$$

Here $p+q=k+2, p \geq 1$, hence $q+2 \leq k+3$. Then the terms of the form $J_{2}$ are all bounded by Lemma 3.2. For the term $J_{1}$, we use Taylor's formula

$$
a(t)-a(z)=\sum_{1 \leq p+q \leq k+1} a_{p q}(z)(t-z)^{p}(\bar{t}-\bar{z})^{q}+O\left(|t-z|^{k+1+\alpha}\right) .
$$

The term corresponding to the remainder in (13) is estimated directly; it has the order $O\left(s^{\alpha-1}\right)$. Now by Lemma 3.2 the estimate $J_{1}(z)=O\left(s^{\alpha-1}\right)$ follows. Theorem is proved.

Finally we include a simple result that applies to $\left[S_{\Omega}, a\right]$ above. 
Proposition 3.3. Let $\Omega \subset \mathbb{C}$ be a bounded domain. Let $k: \Omega \times \Omega \rightarrow \mathbb{C}$ satisfy $|k(z, t)| \leq|z-t|^{\alpha-2}, 0<\alpha<1$. Let $R u(z)=\int_{\Omega} k(z, t) u(t) d^{2} t$. Let $p \geq 1$. Let $p \leq r<\frac{2 p}{2-\alpha p}$ (if $2-\alpha p<0$, then $\left.p \leq r \leq \infty\right)$. Then $R: L^{p}(\Omega) \rightarrow$ $L^{r}(\Omega)$ is a bounded operator, which in particular holds if $r=p+\frac{\alpha}{2}$.

Proof. For completeness we include a proof. Since $\Omega$ is bounded, for $\lambda>-2$ there is a constant $C(\lambda)>0$ such that $\int_{\Omega}|z-t|^{\lambda}\left|d^{2} t\right| \leq C(\lambda)$.

Let $0 \leq c \leq 1, \frac{1}{p}+\frac{1}{q}=1, \frac{1}{r}+\frac{1}{s}+\frac{1}{q}=1$, hence $s=\frac{r p}{r-p}$. By Hölder inequality,

$$
\begin{aligned}
|R u(z)| & =\left|\int_{\Omega} k(z, t) u(t) d^{2} t\right| \leq \int_{\Omega}|u|^{\frac{p}{r}}|k|^{c}|u|^{1-\frac{p}{r}}|k|^{1-c}\left|d^{2} t\right| \\
& \leq\left(\int_{\Omega}|u|^{p}|k|^{c r}\left|d^{2} t\right|\right)^{1 / r}\left(\int_{\Omega}|u|^{p}\left|d^{2} t\right|\right)^{1 / s}\left(\int_{\Omega}|k|^{(1-c) q}\left|d^{2} t\right|\right)^{1 / q}, \\
\|R u\|_{r}^{r} & =\int_{\Omega}|R u(z)|^{r}\left|d^{2} z\right| \leq C((\alpha-2) c r) C((\alpha-2)(1-c) q)^{r / q}\|u\|_{p}^{r},
\end{aligned}
$$

provided that $(\alpha-2) c r>-2$ and $(\alpha-2)(1-c) q>-2$. These conditions yield the desired bounds for $r$.

\section{The operator $S_{\Omega} \bar{S}_{\Omega}$}

The standard approach to the Beltrami type equations (see $[2,13]$ ) involves the Cauchy-Green operator

$$
T_{\Omega} u(z)=\int_{\Omega} \frac{u(t) d^{2} t}{t-z}
$$

for a domain $\Omega \subset \mathbb{C}$. We also consider its modification suitable for solving the Dirichlet problem in the unit disc $\mathbb{D}=\{z \in \mathbb{C}:|z|<1\}$.

$$
T_{1} u(z)=T_{\mathbb{D}} u(z)-\overline{T_{\mathbb{D}} u\left(\bar{z}^{-1}\right)}=\int_{\mathbb{D}} \frac{u(t) d^{2} t}{t-z}+\int_{\mathbb{D}} \frac{z \overline{u(t)} d^{2} t}{1-z \bar{t}} .
$$

Both operators $T_{\Omega}$ and $T_{1}$ solve the $\bar{\partial}$-problem, and $T_{1}$ in addition satisfies the boundary condition $\left.\operatorname{Re} T_{1} u\right|_{b \mathbb{D}}=0$. Consider the operators $S_{\Omega}$ and $S_{1}$, the $\partial$-derivatives of $T_{\Omega}$ and $T_{1}$. Then

$$
S_{1} u(z)=\text { p.v. } \int_{\mathbb{D}} \frac{u(t) d^{2} t}{(t-z)^{2}}+\int_{\mathbb{D}} \frac{\overline{u(t)} d^{2} t}{(1-z \bar{t})^{2}} .
$$


In other words

$$
S_{1} u=S_{\mathbb{D}} u-B \bar{u}, \quad B v(z)=-\int_{\mathbb{D}} \frac{v(t) d^{2} t}{(1-z \bar{t})^{2}} .
$$

Here $B$ is the Bergmann projection in $\mathbb{D}$ (in this notation $d^{2} t<0$ ). It is well known (see $[2,13])$ that $S_{\mathbb{C}}$ and $S_{1}$ are isometries of $L^{2}(\mathbb{C})$ and $L^{2}(\mathbb{D})$ respectively, that is, $S_{\mathbb{C}} \overline{S_{\mathbb{C}}}=I$ and $S_{1} \overline{S_{1}}=I$, here $I=$ id is the identity operator. (Note $S_{\mathbb{C}}^{*}=\overline{S_{\mathbb{C}}}$ and $S_{1}^{*}=\overline{S_{1}}$.) We make the following observation.

Lemma 4.1. $S_{\mathbb{D}} \overline{S_{\mathbb{D}}}=I-B$ and $B S_{\mathbb{D}}+S_{\mathbb{D}} \bar{B}=0$.

Proof. Introduce the conjugation operator $\iota u=\bar{u}$. Then $\iota^{2}=I$. For every operator $P$ we have by definition $\bar{P}=\iota P \iota$ or $\iota P=\bar{P} \iota$, in particular $\bar{\iota}=\iota$. For simplicity put $S=S_{\mathbb{D}}$. Then we write $S_{1}=S-B \iota, \bar{S}_{1}=\bar{S}-\bar{B} \iota$. Using $B^{2}=B$ we obtain

$$
\begin{aligned}
\mathrm{id} & =S_{1} \overline{S_{1}}=(S-B \iota)(\bar{S}-\bar{B} \iota) \\
& =S \bar{S}-B \iota \bar{S}-S \bar{B} \iota+B \iota \bar{B} \iota=(S \bar{S}+B)-(B S+S \bar{B}) \iota .
\end{aligned}
$$

Now by separating linear and anti-linear terms, we obtain the desired relations.

We now consider the operator $B_{\Omega}=I-S_{\Omega} \bar{S}_{\Omega}$ for an arbitrary smooth domain $\Omega \subset \mathbb{C}$. We will see in the next section that $B_{\Omega}$ is related to the Bergmann projection for $\Omega$. Here we only care to what extent $B^{2}=B$ holds for $B_{\Omega}$. Invoke the Cauchy type integral

$$
K_{\Omega} u(z)=\frac{1}{2 \pi i} \int_{b \Omega} \frac{u(t) d t}{t-z}, \quad z \in \Omega .
$$

For $z \in b \Omega$ we interpret $K_{\Omega} u(z)$ as a boundary value of the function $K_{\Omega} u$ in $\Omega$. With some abuse of notation we write $\partial u(z)=\partial_{z} u(z)$ and $\bar{\partial} u(z)=$ $\partial_{\bar{z}} u(z)$. We recall the Cauchy-Green-Pompeiu formula

$$
K_{\Omega}+T_{\Omega} \bar{\partial}=I
$$

The following result is similar to one by Kerzman and Stein [8] who discovered that $K_{\Omega}-K_{\Omega}^{*}$ is a smoothing operator.

Theorem 4.2. Let $\Omega \subset \mathbb{C}$ be a bounded domain of class $C^{k}$, here $k \geq 1$ may be fractional. Then $P=K_{\Omega}+\bar{K}_{\Omega}-I$ is a bounded operator $L^{1}(b \Omega) \rightarrow$ $C^{k-2}(b \Omega)($ if $k \geq 2)$ and $C^{1}(b \Omega) \rightarrow C^{k-1}(b \Omega)$. 
Proof. Observe for $z \in \Omega, t \in b \Omega$

$$
\frac{d t}{t-z}-\frac{d \bar{t}}{\bar{t}-\bar{z}}=\frac{\bar{t}-\bar{z}}{t-z} d_{t}\left(\frac{t-z}{\bar{t}-\bar{z}}\right)=d_{t} \log \left(\frac{t-z}{\bar{t}-\bar{z}}\right)=2 i d_{t} \arg (t-z) .
$$

Then for $z \in \Omega$

$$
\left(K_{\Omega}+\bar{K}_{\Omega}\right) u(z)=\frac{1}{2 \pi i} \int_{b \Omega} u(t)\left(\frac{d t}{t-z}-\frac{d \bar{t}}{\bar{t}-\bar{z}}\right)=\frac{1}{\pi} \int_{b \Omega} u(t) d_{t} \arg (t-z) .
$$

If $z \in b \Omega$, then passing to the limit yields

$$
\left(K_{\Omega}+\bar{K}_{\Omega}\right) u(z)=u(z)+\frac{1}{\pi} \mathrm{p} \cdot \mathrm{v} \cdot \int_{b \Omega} u(t) d_{t} \arg (t-z) .
$$

The only reason for principal value in this integral is the jump of $-\pi$ of $\arg (t-z)$ at $t=z$. Otherwise the integral has a smooth kernel. Indeed, suppose an arc of $b \Omega$ has a parametric equation $\gamma(\tau)=\tau+i \phi(\tau)$ with $\phi \in$ $C^{k}$. Then for $t=\gamma(\tau), z=\gamma\left(\tau_{0}\right)$, we have

$$
d_{t} \arg (t-z)=d \arctan \left(\frac{\phi(\tau)-\phi\left(\tau_{0}\right)}{\tau-\tau_{0}}\right) \in C^{k-2} .
$$

Hence if $u \in L^{1}(b \Omega)$, then $P u \in C^{k-2}(b \Omega)$. If $u \in C^{1}(b \Omega)$, then by integrating by parts $P u \in C^{k-1}(b \Omega)$.

Corollary 4.3. Let $\Omega \subset \mathbb{C}$ be a bounded domain of class $C^{k}$, here $k>2$ is fractional. Then $B_{\Omega}^{2}-B_{\Omega}$ is a bounded operator $L^{p}(\Omega) \rightarrow C^{k-3}(\bar{\Omega})$ (if $p>1, k>3$ ) and $C^{\alpha}(\Omega) \rightarrow C^{k-2}(\bar{\Omega})$ (if $0<\alpha<1, k>2$ ).

Proof. For simplicity of notation we omit the subscripts $\Omega$. We have

$$
\begin{aligned}
K P & =K(K+\bar{K}-I)=K+K \bar{K}-K=K \bar{K}, \\
B & =I-S \bar{S}=I-\partial(T \bar{\partial}) \bar{T}=I-\partial(I-K) \bar{T}=\partial K \bar{T}, \\
B^{2} & =\partial K(\bar{T} \partial) K \bar{T}=\partial K(I-\bar{K}) K \bar{T}=B-\partial K P K \bar{T} .
\end{aligned}
$$

Let $u \in L^{p}(\Omega), p>1$. Then $\bar{T} u \in W^{1, p}(\Omega)$, the trace $\left.\bar{T} u\right|_{b \Omega} \in L^{p}(b \Omega)$, and $K \bar{T} u \in L^{p}(b \Omega)$. By Theorem 4.2, $P K \bar{T} u \in C^{k-2}(b \Omega)$. Since $k$ is fractional, we have $K P K \bar{T} u \in C^{k-2}(\Omega)$, and $\left(B^{2}-B\right) u=-\partial K P K \bar{T} u \in C^{k-3}(\Omega)$, as desired.

Let $u \in C^{\alpha}(\Omega)$. Then $\bar{T} u \in C^{1+\alpha}(\Omega)$ and $K \bar{T} u \in C^{1+\alpha}(\Omega)$. By Theorem 4.2, $P K \bar{T} u \in C^{k-1}(b \Omega)$. Since $k$ is fractional, we have $K P K \bar{T} u \in$ $C^{k-1}(\Omega)$, and $\left(B^{2}-B\right) u \in C^{k-2}(\Omega)$, as desired. 


\section{The Bergman projection}

The main result of this section is an asymptotic formula for the Bergman projection $\mathcal{B}=\mathcal{B}_{\Omega}$ for a smooth bounded simply connected domain $\Omega \subset \mathbb{C}$. We will not need it in the rest of the paper.

Let $H=H(\Omega)$ be the Bergman space of all holomorphic functions of class $L^{2}(\Omega)$. The Bergman projection $\mathcal{B}: L^{2}(\Omega) \rightarrow H$ is the orthogonal projection onto the subspace $H \subset L^{2}(\Omega)$.

Theorem 5.1. Let $\Omega \subset \mathbb{C}$ be a bounded domain of class $C^{k}$, here $k>3$ is fractional. Let $B_{n}=I-(S \bar{S})^{n}, S=S_{\Omega}, n \geq 1$. Then

(i) For all $n \geq 1$, the difference $\mathcal{B}-B_{n}$ is a bounded operator $L^{2}(\Omega) \rightarrow$ $C^{k-3}(\Omega)$.

(ii) If $\Omega$ is simply connected, then $\mathcal{B}=\lim _{n \rightarrow \infty} B_{n}$.

We can compare this result to the one by Kerzman and Stein [8]. Let $\mathcal{S}: L^{2}(b \Omega) \rightarrow H^{2}(b \Omega)$ be the Szegö orthogonal projection, and let $K=K_{\Omega}$ be the Cauchy transform. Kerzman and Stein [8] proved that $\mathcal{S}-K$ is a compact smoothing operator and $\mathcal{S}=K(I-A)^{-1}$, here $A=K-K^{*}$ is a compact smoothing operator. If $\Omega$ is sufficiently close to the disc $\mathbb{D}$, then $\|A\|<1$, and the inverse has an explicit formula $(I-A)^{-1}=\sum_{n=0}^{\infty} A^{n}$. In contrast, our formula $\mathcal{B}=\lim _{n \rightarrow \infty} B_{n}$ holds for every simply connected smooth domain.

We also compare Theorem 5.1(i) with the formula $\mathcal{B}=\partial E \bar{T}$ (Bell [3], page 70$)$. Here $E$ denotes the harmonic extension from $b \Omega$ to $\Omega$. For a general domain, $E$ is not explicit. If we replace $E$ by $K$, then by (16) we obtain the explicit operator $B=B_{1}$, which by Theorem 5.1(i) approximates the Bergman projection $\mathcal{B}$.

Lemma 5.2. The subspace $H \subset L^{2}(\Omega)$ is invariant for $S \bar{S}$, and $\left.S \bar{S}\right|_{H}$ : $H \rightarrow C^{k-3}(\bar{\Omega})$ is bounded.

Proof. By (16), the subspace $H \subset L^{2}(\Omega)$ is invariant for $B=I-S \bar{S}$, hence for $S \bar{S}$. Let $H_{0} \subset H$ consist of such $u \in H$ that for every closed path $\gamma \subset \Omega$, we have $\int_{\gamma} u(z) d z=0$. For $u \in H_{0}$ define $J u(z)=\int_{z_{0}}^{z} u(t) d t$ along a path in $\Omega$. Then for $u \in H_{0}$

$$
\begin{aligned}
S \bar{S} u=\partial(T \bar{\partial})(\bar{T} \partial) J u & =\partial(I-K)(I-\bar{K}) J u \\
& =\partial(I-K-\bar{K}+K \bar{K}) J u=\partial K \bar{K} J u=\partial K P J u
\end{aligned}
$$


Since $u \in L^{2}(\Omega)$, we have $J u \in W^{1,2}(\Omega)$. Then the trace $\left.J u\right|_{b \Omega} \in L^{2}(b \Omega)$. Then by Theorem 4.2, we have $P J u \in C^{k-2}(b \Omega), K P J u \in C^{k-2}(\bar{\Omega})$, and finally $S \bar{S} u=\partial K P J u \in C^{k-3}(\bar{\Omega})$.

If $\Omega$ is simply connected, then $H_{0}=H$, and the proof is complete. Otherwise, $H_{0}$ has a finite dimensional (not necessarily orthogonal) complement $H_{1}$ in $H$ of the form, say

$$
H_{1}=\left\{\sum c_{j}\left(z-z_{j}\right)^{-1}: c_{j} \in \mathbb{C}\right\} .
$$

Here the points $z_{j} \in \mathbb{C} \backslash \Omega$ are fixed - one in each bounded component of $\mathbb{C} \backslash \Omega$. Since $H_{1}$ consists of smooth functions, the operator $\left.S \bar{S}\right|_{H_{1}}: H_{1} \rightarrow$ $C^{k-3}(\bar{\Omega})$ is bounded, hence the desired conclusion.

Lemma 5.3. If $\Omega$ is simply connected, then $\|S \bar{S}\|_{H}<1$.

Proof. Note $\bar{S}=S^{*}$. Since $\left.S \bar{S}\right|_{H}$ is self-adjoint, compact, and $S \bar{S} \geq 0$, it suffices to show that $\left.S \bar{S}\right|_{H}$ does not have the eigenvalue 1 .

Suppose there is $u \in H$ such that $S \bar{S} u=u$. Since $\|S\|_{2} \leq 1$, we have $\|S \bar{S} u\|_{2} \leq\|\bar{S} u\|_{2} \leq\|u\|_{2}$. Since $S \bar{S} u=u$, we have in particular, $\|\bar{S} u\|_{2}=$ $\|u\|_{2}$. On the other hand, $S_{\mathbb{C}}$ is an isometry of $L^{2}(\mathbb{C})$. Hence $\bar{S} u(z)=0$ for $z \notin \Omega$, that is, $\overline{\partial T} u=0$ in $\mathbb{C} \backslash \Omega$.

Since $\bar{T} u$ is antiholomorphic on a connected set $\mathbb{C} \backslash \Omega$ and $\overline{\partial T} u=0$, the function $\bar{T} u=$ const in $\mathbb{C} \backslash \Omega$. In fact $\left.\bar{T} u\right|_{\mathbb{C} \backslash \Omega}=0$ because it vanishes at infinity. By Lemma 5.2, $u \in C^{k-3}(\bar{\Omega})$, hence $\bar{T} u$ is continuous on $\mathbb{C}$.

Since $u$ is holomorphic, $\bar{\partial} \partial \bar{T} u=\bar{\partial} u=0$, that is, $\bar{T} u$ is harmonic in $\Omega$. Since $\left.\bar{T} u\right|_{b \Omega}=0$, we have $\bar{T} u=0$ in $\Omega$. Hence $u=\partial \bar{T} u=0$, and the proof is complete.

Proof of Theorem 5.1. By (16), we have $B\left(L^{2}(\Omega)\right) \subset H$. Since $B$ is self-adjoint, $B\left(H^{\perp}\right)=0$. Indeed, for every $u \in H^{\perp}$ and $v \in L^{2}(\Omega)$, we have $(B u, v)=(u, B v)=0$. Hence, $S \bar{S} H \subset H$ and $\left.S \bar{S}\right|_{H^{\perp}}=I$.

We now compare $B_{n}$ with $\mathcal{B}$ on $H$ and $H^{\perp}$. On $H^{\perp}$ we have $\mathcal{B}\left(H^{\perp}\right)=$ $B_{n}\left(H^{\perp}\right)=0$. On $H$ we have $\left.\left(\mathcal{B}-B_{n}\right)\right|_{H}=\left.(S \bar{S})^{n}\right|_{H}: H \rightarrow C^{k-3}(\bar{\Omega})$, which proves (i).

By Lemma 5.3, $\left.(S \bar{S})^{n}\right|_{H} \rightarrow 0$ as $n \rightarrow \infty$, hence the conclusion (ii).

We realize that if $\Omega$ is not simply connected, then Lemma 5.3 and Theorem 5.1(ii) fail as the following simple example shows.

Example 5.4. Let $0<r<1$ and let $\Omega=\{z: r<|z|<1\}$. Let $u(z)=$ $1 / z$. Then one can find $\bar{T} u(z)=2 \log |z|$. (It is independent of $r$.) Then 
$S \bar{S} u(z)=\partial T \bar{\partial} T u(z)=\partial T \bar{\partial}(2 \log |z|)=\partial T(1 / \bar{z})=\partial(2 \log |z|)=1 / z=u(z)$. Then $B_{n} u=0$, but $\mathcal{B} u=u$, so Lemma 5.3 and Theorem 5.1(ii) fail.

\section{Integral equations with operator $S$}

We now consider the integral equation

$$
u=S(A \bar{u})+b .
$$

Here $u$ and $b$ are $m$-vector functions and $A$ is a $m \times m$ matrix function in a smooth bounded domain $\Omega \subset \mathbb{C} ; m \geq 1, S=S_{\Omega}$. In the future, with some abuse of notation, we omit the parentheses in (17) and similar equations, interpreting $A$ as the operator of multiplication by $A$. We impose the condition $\|A\|_{\infty}<1$. Here $\|A\|_{\infty}$ denotes the maximum of the Euclidean operator norm of $A(z)$ over all $z \in \bar{\Omega}$.

Proposition 6.1. Let $\Omega \subset \mathbb{C}$ be a bounded domain of class $C^{\infty}$. Let $A, b \in$ $C^{k, \alpha}(\Omega), 0<\alpha<1, k \geq 0,\|A\|_{\infty}<1$. Then the Equation (17) has a unique solution $u \in L^{2}(\Omega)$. This solution $u \in C^{k, \alpha}(\Omega)$, and for fixed $A$ the operator $b \mapsto u$ is bounded in $C^{k, \alpha}(\Omega)$

The proof below goes through if $\Omega$ has finite smoothness of class $C^{3, \beta}$ $(0<\beta<1)$ if $k=0$ and $C^{k+2, \alpha}$ if $k \geq 1$.

Proof. The existence of a unique solution $u \in L^{2}(\Omega)$ is standard (see $[2,13]$ ). It follows because $\|S \circ A\|_{2}<1$ as an operator in $L^{2}(\Omega)$.

Iterating (17) yields

$$
u=S A \overline{S A} u+b_{1}, \quad b_{1}=S A \bar{b}+b \in C^{k, \alpha}(\Omega) .
$$

Interchanging $\bar{S}$ and $A$ yields

$$
u=S \bar{S} A \bar{A} u+b_{2}, \quad b_{2}=S[A, \bar{S}] \bar{A} u+b_{1} .
$$

We include the term $S[A, \bar{S}] \bar{A} u$ in $b_{2}$ because by the results of Section 3 the commutator is "better" than $u$. Recall $S \bar{S}=I-B, B=B_{\Omega}$. Since $[P Q, R]=[P, R] Q+P[Q, R]$, both Theorem 3.1 and Proposition 3.3 apply 
to $B$. We put $v=A \bar{A} u$. Then

$$
v=A \bar{A}(v-B v)+b_{3}, \quad b_{3}=A \bar{A} b_{2}
$$

Since $|A|<1$, we have $(I-A \bar{A})^{-1} \in C^{k, \alpha}$, and

$$
v=-A_{0} B v+b_{4}, \quad A_{0}=(I-A \bar{A})^{-1} A \bar{A}, \quad b_{4}=(I-A \bar{A})^{-1} b_{3} .
$$

Applying $B$ and interchanging $B$ and $A_{0}$ yields

$$
\begin{aligned}
& B v=-B A_{0} B v+B b_{4}=-\left(\left[B, A_{0}\right]+A_{0} B\right) B v+B b_{4}=-A_{0} B v+b_{5}, \\
& b_{5}=-\left[B, A_{0}\right] B v+A_{0}\left(B-B^{2}\right) v+B b_{4} .
\end{aligned}
$$

Note that by Corollary 4.3 the term $\left(B-B^{2}\right) v$ is $C^{\infty}$. Also note $(I+$ $\left.A_{0}\right)^{-1}=I-A \bar{A}$ and $A_{0}(I-A \bar{A})=A \bar{A}$. Then

$$
B v=(I-A \bar{A}) b_{5}, \quad v=-A \bar{A} b_{5}+b_{4}, \quad u=S \bar{S} v+b_{2}
$$

As a result, the initial equation implies

$$
u=M u+N b
$$

where $M$ is a smoothing operator with properties described in Theorem 3.1 and Proposition 3.3, and $N$ is a bounded operator in $C^{k, \alpha}$.

We now use (18) for bootstrapping, successively improving the regularity of the solution. Since $u \in L^{p}$, starting from $p=2$, by Proposition 3.3, Mu $\in$ $L^{p+\frac{\alpha}{2}}$, hence by (18) $u \in L^{p+\frac{\alpha}{2}}$. We repeat this argument finitely many times till we get $u \in L^{r}, r>\frac{2}{\alpha}$. Repeating it one more time, by Proposition 3.3 we get $u \in L^{\infty}$. We now repeat it again finitely many times using Theorem 3.1 and get $u \in C^{k, \alpha}$ as desired. Note that the number of times we iterate (18) depends only on $k$ and $\alpha$.

We now consider a similar integral equation in the unit disc, namely

$$
u=S_{1}(A \bar{u})+b
$$

Proposition 6.2. For the Equation (19), Proposition 6.1 holds.

Proof. The argument of the proof is similar to that for Proposition 6.1. The difference is that the results of Section 3 do not directly apply to $\left[S_{1}, A\right]$ 
because $S_{1}$ is not complex linear. Nevertheless we reduce the result to (the proof of) Proposition 6.1. By the definition of $S_{1}$

$$
u=S A \bar{u}-B \bar{A} u+b,
$$

here $S=S_{\mathbb{D}}, B=B_{\mathbb{D}}$. By Lemma 4.1

$$
B u=-S \bar{B} A \bar{u}-B \bar{A} u+B b .
$$

Multiplying by $\bar{A}$ and interchanging $\bar{A}$ and $B$ yields

$$
B \bar{A} u=-\bar{A} S \bar{B} A \bar{u}-\bar{A} B \bar{A} u+b_{1}, \quad b_{1}=-[\bar{A}, B] u+\bar{A} B b .
$$

Then $v=B \bar{A} u$ satisfies the equation $v=-\bar{A} S \bar{v}-\bar{A} v+b_{1}$, which in turn simplifies to

$$
v=A_{1} S \bar{v}+b_{2}, \quad A_{1}=-(I+\bar{A})^{-1} \bar{A}, \quad b_{2}=(I+\bar{A})^{-1} b_{1} .
$$

This equation looks similar to (17), however $\left\|A_{1}\right\|_{\infty}<1$ need not hold, so the equation requires a little more care. Following the beginning of the proof of Proposition 6.1, iterating (20) yields

$$
v=A_{1} S \bar{A}_{1} \bar{S} v+b_{3}, \quad b_{3}=A_{1} S \bar{b}_{2}+b_{2} .
$$

Interchanging $S$ and $\bar{A}_{1}$ yields

$$
v=A_{1} \bar{A}_{1} S \bar{S} v+b_{4}, \quad b_{4}=A_{1}\left[S, \bar{A}_{1}\right] \bar{S} v+b_{3} .
$$

Since $v=B \bar{A} u$ and $B^{2}=B$, we have $S \bar{S} v=v-B v=0$. Hence $v=b_{4}$, and the original equation takes the form

$$
u=S A \bar{u}+b_{5}, \quad b_{5}=-b_{4}+b,
$$

which is the subject of Proposition 6.1. By bootstrapping we obtain $u \in$ $C^{k, \alpha}(\mathbb{D})$.

\section{Dirichlet problem}

We consider the Dirichlet problem for an elliptic equation

$$
f_{\bar{z}}=a(z) f_{z}+b(z) \bar{f}_{\bar{z}}+c(z) .
$$


In the scalar case the ellipticity means that either $|a|+|b|<1$ or ||$a|-| b||>$ 1 ; the two cases are related by the interchange $f \leftrightarrow \bar{f}$. We restrict to the former case. Our main result is the following.

Theorem 7.1. Let $\Omega \subset \mathbb{C}$ be a simply connected domain of class $C^{k+1, \alpha}$, $k \geq 0,0<\alpha<1$. Let $a, b, c \in C^{k, \alpha}(\Omega), f_{0} \in C^{k+1, \alpha}(b \Omega, \mathbb{R}),|a|+|b| \leq a_{0}<$ 1 , for some constant $a_{0}$. Then the scalar equation (21) with boundary condition $\left.\operatorname{Re} f\right|_{b \Omega}=f_{0}$ has a unique solution in the Sobolev class $W^{1,2}(\Omega)$. This solution $f \in C^{k+1, \alpha}(\Omega)$, and for fixed $a$ and $b$ the map $\left(c, f_{0}\right) \mapsto f$ is a bounded operator $C^{k, \alpha}(\Omega) \times C^{k+1, \alpha}(b \Omega, \mathbb{R}) \rightarrow C^{k+1, \alpha}(\Omega)$.

For simplicity we assume that $\Omega$ is simply connected because our method involves reduction to the unit disc. Thus we begin the proof with several reductions.

Lemma 7.2. It suffices to prove Theorem 7.1 for $f_{0}=0$ and $\Omega=\mathbb{D}$, the unit disc.

Proof. To reduce to $f_{0}=0$, we fix $f_{1} \in C^{k+1, \alpha}(\Omega)$ satisfying $\left.\operatorname{Re} f_{1}\right|_{b \Omega}=f_{0}$. Then for the new unknown $\tilde{f}=f-f_{1}$, the equation will have a form similar to the original one, and the boundary condition will turn into $\left.\operatorname{Re} \tilde{f}\right|_{b \Omega}=0$.

To reduce to $\Omega=\mathbb{D}$ we can introduce a new independent variable $\zeta=$ $\psi(z)$, so that $\psi: \bar{\Omega} \rightarrow \overline{\mathbb{D}}$ is a $C^{k+1, \alpha}$ diffeomorphism with positive Jacobian. The equation will preserve its form and boundary conditions. Moreover, if $a=0$ or $b=0$, then by choosing a conformal map $\psi$ this condition can be preserved also.

Lemma 7.3. It suffices to prove Theorem 7.1 for $a=0$.

Proof. We change the independent variable by a Beltrami homeomorphism $\psi: \mathbb{D} \rightarrow \mathbb{D}$ of the equation

$$
\psi_{\bar{z}}=\mu(z) \psi_{z}
$$

The Beltrami coefficient $\mu$ will be determined later. The Equation (21) will take the form

$$
g_{\bar{\zeta}}=\tilde{a} g_{\zeta}+\tilde{b} \bar{g}_{\bar{\zeta}}+\tilde{c}
$$

here $g=f \circ \psi^{-1}$. We write $\zeta=\psi(z)$. By straightforward calculations we now find the new coefficients. We have

$$
g_{\bar{z}}=g_{\zeta} \psi_{\bar{z}}+g_{\bar{\zeta}} \bar{\psi}_{\bar{z}}=a\left(g_{\zeta} \psi_{z}+g_{\bar{\zeta}} \bar{\psi}_{z}\right)+b\left(\bar{g}_{\bar{\zeta}} \bar{\psi}_{\bar{z}}+\bar{g}_{\zeta} \psi_{\bar{z}}\right)+c .
$$


By (22) we obtain

$$
\bar{\psi}_{\bar{z}}(1-a \bar{\mu}) g_{\bar{\zeta}}-\psi_{z} b \mu \bar{g}_{\zeta}=\psi_{z}(a-\mu) g_{\zeta}+\bar{\psi}_{\bar{z}} b \bar{g}_{\bar{\zeta}}+c
$$

We solve (24) together with its conjugate as a system of two equations with the two unknowns $g_{\bar{\zeta}}$ and $\bar{g}_{\zeta}$. By ellipticity it has a unique solution. In particular,

$$
\tilde{a}=\frac{\psi_{z}}{\bar{\psi}_{\bar{z}}} \frac{(a-\mu)(1-\bar{a} \mu)+|b|^{2} \mu}{|1-\bar{a} \mu|^{2}-|b \mu|^{2}} .
$$

The equation $\tilde{a}=0$ turns into a quadratic equation on $\mu$ of the form

$$
\bar{a} \mu^{2}-\left(1+|a|^{2}-|b|^{2}\right) \mu+a=0 .
$$

Due to $|a|+|b|<1$, the equation has two distinct solutions $\mu_{1}, \mu_{2},\left|\mu_{1} \mu_{2}\right|=$ 1 . We chose $\mu=\mu_{1}$, the one with smaller modulus. (If $a(z)=0$ at some $z$, then $\mu_{1}(z)=0, \mu_{2}(z)=\infty$.) It is easy to see $\mu \in C^{k, \alpha}$ and $\|\mu\|_{\infty}<1$. Hence, the homeomorphism $\psi \in C^{k+1, \alpha}$, the new coefficients in (23) are in $C^{k, \alpha}$, and $\tilde{a}=0$.

Slightly changing notation, we now consider the equation

$$
f_{\bar{z}}=A(z) \bar{f}_{\bar{z}}+b(z)
$$

Here for the sake of generality, $f$ and $b$ are $m$-vectors and $A$ is a $m \times m$ matrix, $m \geq 1$. To complete the proof of Theorem 7.1 we need the following result only in the case $\Omega=\mathbb{D}, f_{0}=0$.

Theorem 7.4. Let $\Omega \subset \mathbb{C}$ be a simply connected domain of class $C^{k+1, \alpha}$, $k \geq 0,0<\alpha<1$. Let $A, b \in C^{k, \alpha}(\Omega), f_{0} \in C^{k+1, \alpha}(b \Omega),\|A\|_{\infty}<1$. Then the Equation (27) with boundary condition $\left.\operatorname{Re} f\right|_{b \Omega}=f_{0}$ has a unique solution in the Sobolev class $W^{1,2}(\Omega)$. This solution $f \in C^{k+1, \alpha}(\Omega)$, and for fixed $A$ the map $\left(b, f_{0}\right) \mapsto f$ is a bounded operator $C^{k, \alpha}(\Omega) \times C^{k+1, \alpha}(b \Omega) \rightarrow C^{k+1, \alpha}(\Omega)$.

Proof. By Lemma 7.2 it suffices to prove the result for $\Omega=\mathbb{D}$ and $f_{0}=0$. For $f \in W^{1,2}(\mathbb{D})$, the Equation $(27)$ with boundary conditions $\left.\operatorname{Re} f\right|_{b \Omega}=0$ 
is equivalent to

$$
f=T_{1}\left(A \bar{f}_{\bar{z}}+b\right) .
$$

If this equation has a solution in $W^{1,2}(\mathbb{D})$, then $u=f_{z}$ satisfies the equation

$$
u=S_{1}(A \bar{u}+b)
$$

The latter by Proposition 6.2 has a unique solution $u \in L^{2}(\mathbb{D})$. This solution is in $C^{k, \alpha}(\mathbb{D})$. Then $f:=T_{1}(A \bar{u}+b) \in C^{k+1, \alpha}(\mathbb{D})$ satisfies $(28)$ because $f_{z}=$ $S_{1}(A \bar{u}+b)=u$.

\section{Inverting $f \mapsto f-T\left(a f_{z}+b \bar{f}_{\bar{z}}\right)$ in Lipschitz spaces}

Let $\Omega \subset \mathbb{C}$ be a bounded domain. We consider the integral equation

$$
f=T\left(a f_{z}+b \bar{f}_{\bar{z}}\right)+c
$$

here $a, b$, and $c$ are given functions in $\Omega, f$ is unknown, and $T=T_{\Omega}$. Solving this equation may be regarded as inverting the operator $f \mapsto f-T\left(a f_{z}+\right.$ $\left.b \bar{f}_{\bar{z}}\right)$. Note that the solution satisfies the boundary condition $K f=K c$ because $K T=0$, here $K=K_{\Omega}$ is the Cauchy type integral. To make the problem look similar to the one in the previous section, we again consider the equation

$$
f_{\bar{z}}=a(z) f_{z}+b(z) \bar{f}_{\bar{z}}+c(z)
$$

with boundary condition $K f=K f_{0}$ for a given function $f_{0}$ on $b \Omega$. Our main result in the scalar case is the following.

Theorem 8.1. Let $\Omega \subset \mathbb{C}$ be a domain of class $C^{k+1, \alpha}, k \geq 0,0<\alpha<1$. Let $a, b, c \in C^{k, \alpha}(\Omega), f_{0} \in C^{k+1, \alpha}(b \Omega),|a|+|b| \leq a_{0}<1$, for some constant $a_{0}$. Then the scalar equation (29) with boundary condition $K f=K f_{0}$ has a unique solution in the Sobolev class $W^{1,2}(\Omega)$. This solution $f \in C^{k+1, \alpha}(\Omega)$, and for fixed $a$ and $b$, the map $\left(c, f_{0}\right) \mapsto f$ is a bounded operator $C^{k, \alpha}(\Omega) \times$ $C^{k+1, \alpha}(b \Omega) \rightarrow C^{k+1, \alpha}(\Omega)$.

We again begin with reductions. Note that $\Omega$ need not be simply connected, so instead of the unit disc, we reduce to a domain of class $C^{\infty}$.

Lemma 8.2. It suffices to prove Theorem 8.1 for $f_{0}=0$ and $\Omega$ of class $C^{\infty}$. 
Proof. To deduce to $K f=0$, we replace $f$ by $f-K f_{0}$. To reduce to a $C^{\infty}$-smooth domain $\Omega_{0}$ we again introduce a new independent variable $\zeta=$ $\psi(z)$ by a $C^{k+1, \alpha}$ diffeomorphism $\psi: \bar{\Omega} \rightarrow \bar{\Omega}_{0}$. To preserve the boundary condition $K f=0$, we first choose a conformal map $\psi: \mathbb{C} \backslash \bar{\Omega} \rightarrow \mathbb{C} \backslash \bar{\Omega}_{0}$, and then extend it $C^{k+1, \alpha}$-smoothly to $\Omega$. (This procedure, however, will not preserve the conditions $a=0$ or $b=0$ if they take place.)

In contrast to the proof of Theorem 7.1, the reduction to $a=0$ is not straightforward because a Beltrami homeomorphism (22) does not preserve the boundary condition. Furthermore, the derivative of $\psi$ enters the boundary condition resulting in a loss of one derivative. We reduce to the case, in which $a$ is small.

Lemma 8.3. Under assumptions of Theorem 8.1, let $f_{0}=0$ and let $\Omega$ be $C^{\infty}$-smooth. Let $\epsilon>0$. There exists a $C^{\infty}$ diffeomorphism $\psi: \mathbb{C} \rightarrow \mathbb{C}$ that transforms the Equation (29) in $\Omega$ into the Equation (23) in $\Omega_{0}=\psi(\Omega)$, in which $\|\tilde{a}\|_{C^{k, \alpha}}<\epsilon$. The boundary condition $K_{\Omega} f=0$ transforms into $K_{\Omega_{0}} g=J g$, here $g=f \circ \psi^{-1}$ and $J: L^{2}\left(b \Omega_{0}\right) \rightarrow C^{\infty}\left(b \Omega_{0}\right)$ is a smoothing operator. Moreover, $\|\psi\|_{C^{k+1, \alpha}}$ is bounded by a constant independent of $\epsilon$.

Proof. Let $C^{\infty}$-smooth functions $a_{0}$ and $b_{0}$ be close to $a_{0}$ and $b_{0}$ in $C^{k, \alpha}(\Omega)$. We find $\mu_{0}$ by solving (26) using $a_{0}$ and $b_{0}$ instead of $a$ and $b$. We assume that $\mu_{0}$ is extended to the whole plane. Following the proof of Lemma 7.3 we make a substitution by a global Beltrami homeomorphism of the Equation (22) with $\mu_{0}$ instead of $\mu$. If $a_{0}$ and $b_{0}$ are sufficiently close to $a$ and $b$, then by (25) the coefficient $\tilde{a}$ in (23), satisfies $\|\tilde{a}\|_{C^{k, \alpha}}<\epsilon$.

We now find out how the substitution affects the boundary condition $K_{\Omega} f=0$. Let $\rho$ be a defining function of $b \Omega_{0}$ with $d \rho \neq 0$ in a neighborhood of $b \Omega_{0}$. Then on $b \Omega_{0}$ we have $\rho_{\zeta} d \zeta+\rho_{\bar{\zeta}} d \bar{\zeta}=0$. Let $\zeta_{0} \in \Omega_{0}$ be sufficiently close to $b \Omega_{0}$. With some abuse of notation we write $z(\zeta)=\psi^{-1}(\zeta), z=z(\zeta)$, $z_{0}=z\left(\zeta_{0}\right)$, etc. We have

$$
\begin{aligned}
0 & =K_{\Omega} f\left(z_{0}\right)=\frac{1}{2 \pi i} \int_{b \Omega} \frac{f(z) d z}{z-z_{0}}=\frac{1}{2 \pi i} \int_{b \Omega_{0}} \frac{g(\zeta)\left(z_{\zeta} d \zeta+z_{\bar{\zeta}} d \bar{\zeta}\right)}{z-z_{0}} \\
& =K_{\Omega_{0}} g\left(\zeta_{0}\right)-J g\left(\zeta_{0}\right), \\
J g\left(\zeta_{0}\right) & =\frac{1}{2 \pi i} \int_{b \Omega_{0}}\left(\frac{1}{\zeta-\zeta_{0}}-\frac{z_{\zeta}-\rho_{\bar{\zeta}}^{-1} \rho_{\zeta} z_{\bar{\zeta}}}{z-z_{0}}\right) g(\zeta) d \zeta .
\end{aligned}
$$


To understand the last integral we introduce

$$
\begin{aligned}
& \Phi\left(\zeta, \zeta_{0}\right)=z(\zeta)-z\left(\zeta_{0}\right)-z_{\zeta}(\zeta)\left(\zeta-\zeta_{0}\right)-z_{\bar{\zeta}}(\zeta) \overline{\left(\zeta-\zeta_{0}\right)} \\
& \phi\left(\zeta, \zeta_{0}\right)=\rho(\zeta)-\rho\left(\zeta_{0}\right)-\rho_{\zeta}(\zeta)\left(\zeta-\zeta_{0}\right)-\rho_{\bar{\zeta}}(\zeta) \overline{\left(\zeta-\zeta_{0}\right)}
\end{aligned}
$$

We will use the last formula for $\zeta \in b \Omega_{0}$, so we will have $\rho(\zeta)=0$. Then $J=J_{1}+J_{2}$, here

$$
\begin{aligned}
J_{1} g\left(\zeta_{0}\right) & =\frac{1}{2 \pi i} \int_{b \Omega_{0}} \frac{\Phi\left(\zeta, \zeta_{0}\right)-\rho_{\bar{\zeta}}^{-1} z_{\bar{\zeta}} \phi\left(\zeta, \zeta_{0}\right)}{\left(\zeta-\zeta_{0}\right)\left(z-z_{0}\right)} g(\zeta) d \zeta \\
J_{2} g\left(\zeta_{0}\right) & =\frac{-\rho\left(\zeta_{0}\right)}{2 \pi i} \int_{b \Omega_{0}} \frac{\rho_{\bar{\zeta}}^{-1} z_{\bar{\zeta}} g(\zeta) d \zeta}{\left(\zeta-\zeta_{0}\right)\left(z-z_{0}\right)} .
\end{aligned}
$$

Since $\Phi\left(\zeta, \zeta_{0}\right)=O\left(\left|\zeta-\zeta_{0}\right|^{2}\right)$ and $\phi\left(\zeta, \zeta_{0}\right)=O\left(\left|\zeta-\zeta_{0}\right|^{2}\right)$, the kernel of the integral $J_{1}$ is $C^{\infty}$-smooth. Hence $J_{1}$ is a smoothing operator. The integral $J_{2}$ reduces to the integral $K_{2}^{0}$ introduced in the proof of Lemma 3.2 by (10). Since $g \in C^{k, \alpha}\left(\Omega_{0}\right) \subset C^{\alpha}\left(\Omega_{0}\right)$, the argument in the proof of that lemma implies $J_{2} g\left(\zeta_{0}\right)=O\left(\left|\rho\left(\zeta_{0}\right)\right|^{\alpha}\right)$. This estimate means that $J_{2} g$ has zero boundary values on $b \Omega_{0}$, and $J$ reduces to the smoothing operator $J_{1}$.

Finally, the $C^{k+1, \alpha}$ norm of $\psi$ depends only on $C^{k, \alpha}$ norm of $\mu_{0}$ (see $[2,13])$, which in turn depends only on $C^{k, \alpha}$ norms of $a$ and $b$, hence the last assertion in the lemma will hold automatically. The lemma is proved.

We again state a special case of Theorem 8.1 in a vector from. Slightly changing notation, we now consider the equation

$$
f_{\bar{z}}=A_{1}(z) f_{z}+A_{2}(z) \bar{f}_{\bar{z}}+b(z) .
$$

Here $f$ and $b$ are $m$-vectors and $A_{1}$ and $A_{2}$ are $m \times m$ matrices, $m \geq 1$. To complete the proof of Theorem 8.1 it suffices to prove the following result.

Theorem 8.4. Let $\Omega \subset \mathbb{C}$ be a $C^{\infty}$-smooth domain. Let $A_{2}, b \in C^{k, \alpha}(\Omega)$, $k \geq 0,0<\alpha<1$. Suppose $\left\|A_{2}\right\|_{\infty}<1$. Then there exists $\epsilon>0$ such that if $A_{1} \in C^{k, \alpha}(\Omega)$ and $\left\|A_{1}\right\|_{C^{k, \alpha}(\Omega)}<\epsilon$, then for every $f_{0} \in C^{k+1, \alpha}(b \Omega)$, the Equation (30) with boundary condition $K_{\Omega} f=K_{\Omega} f_{0}$ has a unique solution in the Sobolev class $W^{1,2}(\Omega)$. This solution $f \in C^{k+1, \alpha}(\Omega)$, and for fixed $A_{1}$ and $A_{2}$, the map $\left(b, f_{0}\right) \mapsto f$ is a bounded operator $C^{k, \alpha}(\Omega) \times C^{k+1, \alpha}(b \Omega) \rightarrow$ $C^{k+1, \alpha}(\Omega)$.

Proof. By Lemma 8.2 we assume $f_{0}=0$. Let $T=T_{\Omega}$ and $S=S_{\Omega}$. For $f \in$ $W^{1,2}(\Omega)$, the Equation (30) with boundary conditions $K_{\Omega} f=0$ is equivalent 
to

$$
f=T\left(A_{1}(z) f_{z}+A_{2}(z) \bar{f}_{\bar{z}}+b\right) .
$$

If this equation has a solution in $W^{1,2}(\Omega)$, then $u=f_{z}$ satisfies the equation

$$
u=S\left(A_{1} u+A_{2} \bar{u}+b\right) .
$$

If $A_{1}$ is small, then of course (32) has a unique solution $u \in L^{2}(\Omega)$. By the proof of Proposition 6.1 the Equation (32) implies

$$
u=M u+N S\left(A_{1} u+b\right) .
$$

Since the operator $N S$ is bounded in $C^{k, \alpha}(\Omega)$, we choose $0<\epsilon<\|N S\|_{C^{k, \alpha}(\Omega)}^{-1}$. Then if $\left\|A_{1}\right\|_{C^{k, \alpha}(\Omega)}<\epsilon$, then $\left(I-N S A_{1}\right)^{-1}$ is bounded in $C^{k, \alpha}(\Omega)$ and $L^{2}(\Omega)$, and

$$
u=M_{1} u+N_{1} b, \quad M_{1}=\left(I-N S A_{1}\right)^{-1} M, \quad N_{1}=\left(I-N S A_{1}\right)^{-1} N S .
$$

By bootstrapping $u \in C^{k, \alpha}(\Omega)$. Then $f:=T\left(A_{1} u+A_{2} \bar{u}+b\right) \in C^{k+1, \alpha}(\Omega)$ satisfies (30) because $f_{z}=S\left(A_{1} u+A_{2} \bar{u}+b\right)=u$.

Proof of Theorem 8.1. There is a unique solution $f \in W^{1,2}(\Omega)$ of $(29)$ with $K_{\Omega} f=0$. Indeed, as we argued before, since $|a|+|b| \leq a_{0}<1$, there is a unique $u \in L^{2}(\Omega)$ satisfying $u=S(a u+b \bar{u}+c)$. Then $f=T(a u+b \bar{u}+c)$. By Lemma 8.3, after the substitution $\zeta=\psi(z)$, the function $g=f \circ \psi^{-1}$ satisfies (23) with small $\|\tilde{a}\|_{C^{k+1, \alpha}}$ and $K_{\Omega_{0}} g=J g$. Since $K_{\Omega} f=0$, of course $J g$ is holomorphic. Since $J g$ is $C^{\infty}$, by Theorem 8.4 we have $g \in C^{k+1, \alpha}\left(\Omega_{0}\right)$. Hence $f \in C^{k+1, \alpha}(\Omega)$.

In conclusion we point out that Theorem 8.4 answers a question raised in [4]. Let $A$ be a $m \times m$ matrix function of class $C^{k, \alpha}(\Omega), k \geq 0,0<\alpha<1$, in a $C^{\infty}$-smooth bounded domain $\Omega \subset \mathbb{C},\|A\|_{\infty}<1, m \geq 2$. The question from [4] (Problem B) reduces to asking whether the operator $f \mapsto f-T_{\Omega}(A \overline{\partial f})$ has a bounded inverse in $C^{k+1, \alpha}(\Omega)$. The affirmative answer is given by Theorem 8.4 with $A_{1}=0, A_{2}=A$. The authors also raise a similar question (Problem A) for the operator $f \mapsto f-T_{\Omega}(A \partial f)$. However, in this paper we are able to treat this question only in the scalar case (Theorem 8.1).

\section{Acknowledgements}

I wish to thank Tadeusz Iwaniec for his letter with a sketch of the proof of Theorem 7.1 for the case $k=0, b=0$ and the case $k \geq 1$. I am also grateful 
to Steve Bell for discussions on the Bergman projection. Finally, I thank Elias Stein for answering my inquiry regarding singular integrals. This work was partially supported by a grant from the Simons Foundation.

\section{References}

[1] S. Agmon, A. Douglis and L. Nirenberg, Estimates near the boundary for solutions of elliptic partial differential equations satisfying general boundary conditions. I, Comm. Pure Appl. Math. 12 (1959), 623-727.

[2] K. Astala, T. Iwaniec, and G. Martin, Elliptic partial differential equations and quasiconformal mappings in the plane, Princeton Mathematical Series, 48, Princeton, 2009, xviii+677 pp.

[3] S. Bell, The Cauchy transform, potential theory, and conformal mapping, CRC Press, Boca Raton, FL, 1992. x+149 pp.

[4] F. Bertrand, X. Gong, and J.-P. Rosay, Common boundary values of holomorphic functions for two-sided complex structures, arXiv: 1008.1234.

[5] A.-P. Calderón, Commutators of singular integral operators, Proc. Nat. Acad. Sci. U.S.A. 53 (1965), 1092-1099.

[6] R. R. Coifman, R. Rochberg, and G. Weiss, Factorization theorems for Hardy spaces in several variables, Ann. of Math. (2) 103 (1976), 611635 .

[7] S. Ivashkovich and J.-P. Rosay, Schwarz-type lemmas for $\bar{\partial}$-inequalities and complete hyperbolicity of almost complex manifolds, Ann. Inst. Fourier 54 (2004), 2387-2435.

[8] N. Kerzman and E. M. Stein, The Cauchy kernel, the Szegö kernel, and the Riemann mapping function, Math. Ann. 236 (1978), 85-93.

[9] A. Nijenhuis and W. Woolf, Some integration problems in almostcomplex and complex manifolds, Ann. of Math. (2) 77 (1963), 429-484.

[10] S. Prössdorf, Some classes of singular equations, North-Holland Publishing Co., Amsterdam-New York, 1978, xiv+417 pp.

[11] J. Schauder, Über lineare elliptische Differentialgleichungen zweiter Ordnung, Math. Z. 38 (1934), 257-282.

[12] A. Sukhov and A. Tumanov, Boundary value problems and J-complex curves, Complex Var. Elliptic Equ. 58 (2013), 1549-1557. 
[13] I. N. Vekua, Generalized analytic functions, Moscow, 1959; English translation: Pergamon Press, London, 1962.

Department of Mathematics, University of Illinois

1409 West Green Street, Urbana, IL 61801, USA

E-mail address: tumanov@illinois.edu

ReCeived July 9, 2014 